10.1117/2.1200909.1796

\title{
Assess DNA functionality in harsh environments
}

Todd Holden, Eric Cheung, George Tremberger Jr., David Lieberman, and Tak Cheung

Bioinformatic techniques enable identification of extremophile life forms well adapted to extraterrestrial environments such as Mars.

When exposed to adverse conditions well in excess of those that might typically be found on Earth, many extremophilic microorganisms-such as (single-celled) halophilic archaea, which thrive in high concentrations of salt-initiate a variety of repair mechanisms that enable them to flourish. ${ }^{1}$ These processes may include correction of errors in their DNA caused by irradiation and strengthening of a radiation-protection shield. The genes associated with these responses possess features that allow the organism to tolerate and evolve in extreme environments. A range of methods have recently been used to describe the nucleotide content and structure of repair-gene sequences of terrestrial bacteria and archaea. ${ }^{2}$ The resulting analyses may cast light on the adaptability of extremophiles equipped with similar repair mechanisms in extraterrestrial environments.

Mars provides one environment that may sustain halophiles, since the salt content of the soils would allow them access to the minerals needed for protection from UV exposure. Therefore, we studied the nucleotide sequences of several DNA repair genes of Halobacterium sp. NRC-1 to characterize their functionality and potentially detect evidence of possible selection pressure. We computed several measures that have been shown to correlate with functionality, including sequence-fluctuation fractal dimension, sequence Shannon dinucleotide entropy, and CpG (cytosine-phosphate-guanine) pair frequency.,

We can measure the complexity of a series using fractaldimension analysis. ${ }^{5}$ The technique is based on Higuchi's algorithm, which compares relative changes in a sequence as a function of scale (see Figure 1). The atomic-number equivalent of each nucleotide is used to generate the numerical series necessary to calculate the fractal dimension. Shannon entropy reflects the level of functional constraints acting on the gene ${ }^{6}$ and is used as a measure of sequence variability. $\mathrm{CpG}$ pair frequencies are statistical measures that reflect levels of gene regulation.

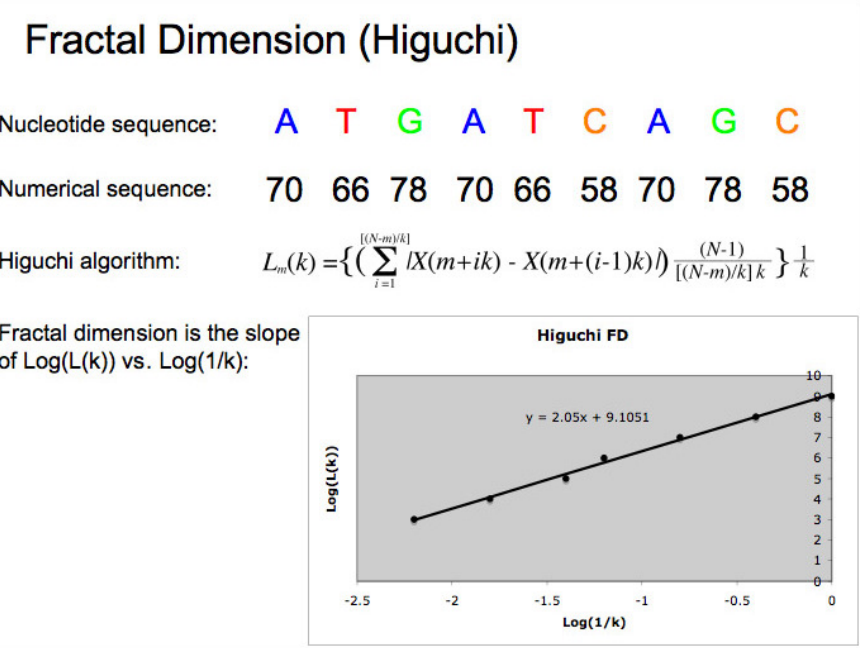

Figure 1. The level of complexity in a nucleotide sequence can be evaluated using the Higuchi method for determining the fractal dimension (FD) of a numerical series. In the Higuchi algorithm, a numerical sequence with $N$ elements, $X(i)$, is analyzed and an average difference, $L(k)$, for sequence elements $k$ steps apart is calculated. $m$ : index of starting element $(m=1,2, \ldots, k-1)$.

We observed correlations among these three measures in the Halobacterium repair genes, suggesting that their sequences have been under selection pressure and can adjust their functionality (see Figures 2 and 3). For instance, we found that the fractal dimensions of the Halobacterium repair genes were relatively high and comparable to those of radiation-resistant Deinococcus radiodurans. Overall, our study supports the use of fractal dimension, Shannon di-nucleotide entropy, and CpG fraction in assessing a microorganism's viability in extreme environments.

By testing these correlations among the repair genes of Halobacterium and other terrestrial extremophiles, we may discover the genetic machinery required for adaptation in harsh environments such as that on Mars. Our results could help

Continued on next page 


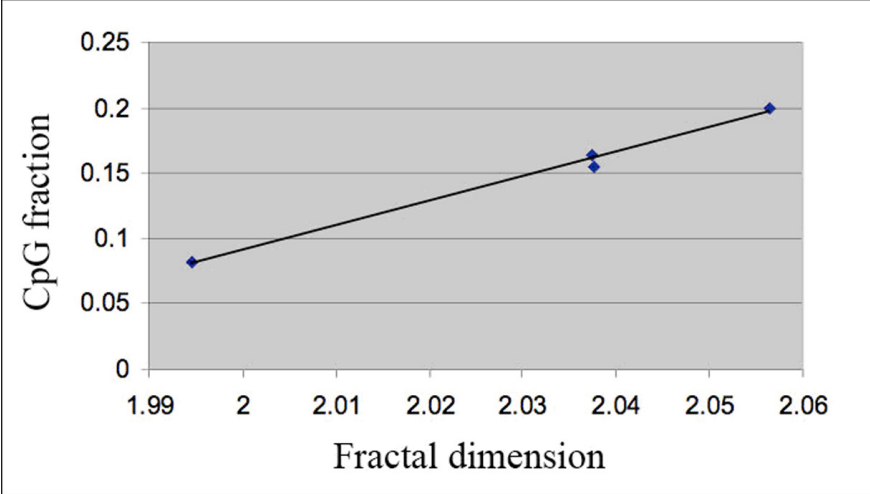

Figure 2. The fractal dimension of Halobacterium NRC-1 repair genes correlates with $C p G$ fraction.

guide development of detection methods such as chiral spectropolarization of biomolecules ${ }^{7}$ and Raman spectroscopy of the carotenoid compounds. ${ }^{8}$ Archaea containing similar genes as Earth's radiation-hardened halophiles may have existed on early Mars and may still be present below its surface. Direct evidence may be found in the salt-rich soils on Mars.

We are currently working to extend our characterization to as many extremophiles as possible to observe trends in highly specialized DNA, including ongoing analysis of psychrophilic methanogens such as Methanococcoides burtonii and Methanosarcina barkeri. In the long term, we seek to include more bioinformatic measures into a powerful multidimensional DNA classification. In addition, we are researching these and other characteristics for possible use in making determination of a DNA segment's biological function ab initio.

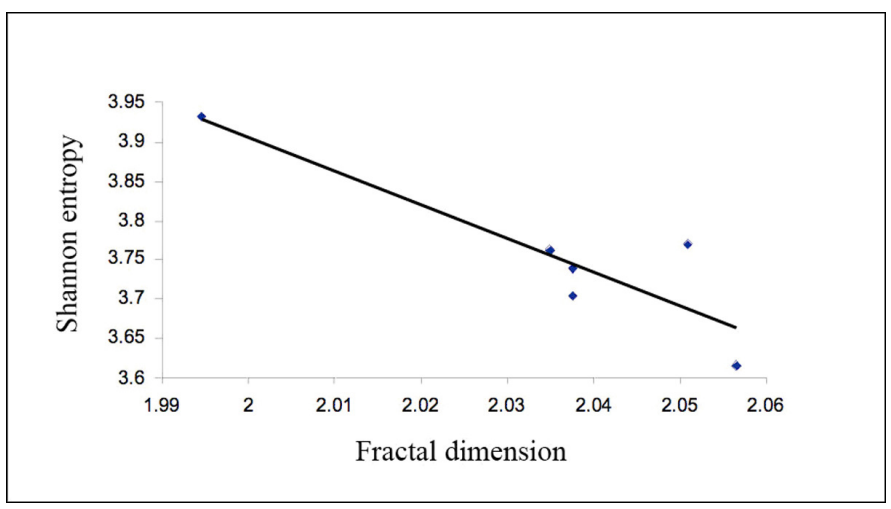

Figure 3. The Shannon entropy of Halobacterium NRC-1 repair genes correlates with fractal dimension.

\section{Author Information}

Todd Holden, Eric Cheung, George Tremberger Jr., David Lieberman, and Tak Cheung

Department of Physics

Queensborough Community College

City University of New York

Bayside, NY

\section{References}

1. L. C. DeVeaux, J. A. Muller, J. Smith, J. Petrisko, D. P. Wells, and S. DasSarmac, Extremely radiation-resistant mutants of a halophilic archaeon with increased singlestranded DNA-binding protein (RPA) gene expression, Radiat. Res. 168, pp. 507-514, 2007.

2. T. Holden, R. Subramaniam, R. Sullivan, E. Cheung, C. Schneider, G. Tremberger Jr., A. Flamholz, D. H. Lieberman, and T. D. Cheung, ATCG nucleotide fluctuation of Deinococcus radiodurans radiation genes, Proc. SPIE 6694, p. 669417,2007 . doi: $10.1117 / 12.732283$

3. G. Tremberger Jr., T. Holden, E. Cheung, R. Subramaniam, R. Sullivan, P. Schneider, A. Flamholz, P. Marchese, D. Lieberman, and T. Cheung, Nucleotide fluctuation of the RecA repair gene in Siberian permafrost Psychrobacter cryohalolentis K5, Proc. SPIE 7097, p. 70970U, 2008. doi:10.1117/12.794390

4. T. Holden, G. Tremberger Jr., E. Cheung, R. Subramaniam, R. Sullivan, P. Schneider, A. Flamholz, D. Lieberman, and T. Cheung, Marfan syndrome exon $C p G$ percentage and fractal dimension, Proc. 2nd IEEE Int'l Conf. Bioinform. Biomed. Eng. (ICBBE), pp. 482-485, 2008.

5. T. Higuchi, Approach to an irregular time series on the basis of fractal theory, Physica D 31, pp. 277-283, 1988.

6. D. V. Parkhomchuk, Di-nucleotide entropy as a measure of genomic sequence functionality, 2006. arXiv:q-bio/0611059

7. W. B. Sparks, J. Hough, T. A. Germer, F. Chen, S. DasSarma, P. DasSarma, F. T. Robb, N. Manset, L. Kolokolova, N. Reid, F. D. Macchetto, and W. Martin, Detection of circular polarization in light scattered from photosynthetic microbes, Proc. Nat'1 Acad. Sci. USA 106, pp. 7816-7821, 2009.

8. S. Fendrihan, M. Musso, and H. Stan-Lotter, Raman spectroscopy as a potential method for the detection of extremely halophilic archaea embedded in halite in terrestrial and possibly extraterrestrial samples, J. Raman Spectrosc. In press. Published online: 10 July 2009, doi:10.1002/jrs.2357 